\title{
High-resolution UVES/VLT spectra of white dwarfs observed for the ESO SN la progenitor survey (SPY). I. ${ }^{\star, \star \star}$
}

\author{
D. Koester ${ }^{1}$, R. Napiwotzki ${ }^{2}$, N. Christlieb ${ }^{3}$, H. Drechsel ${ }^{2}$, H.-J. Hagen ${ }^{3}$, U. Heber ${ }^{2}$, D. Homeier ${ }^{1}$, C. Karl ${ }^{2}$, \\ B. Leibundgut ${ }^{4}$, S. Moehler ${ }^{2}$, G. Nelemans ${ }^{5}$, E.-M. Pauli ${ }^{2}$, D. Reimers ${ }^{3}$, A. Renzini ${ }^{4}$, and L. Yungelson ${ }^{6}$ \\ 1 Institut für Theoretische Physik und Astrophysik, Universität Kiel, 24098 Kiel, Germany \\ 2 Dr.-Remeis-Sternwarte, Astronomisches Institut der Universität Erlangen-Nürnberg, Sternwartstr. 7, \\ 96049 Bamberg, Germany \\ 3 Hamburger Sternwarte, Universität Hamburg, Gojenbergsweg 112, 21029 Hamburg, Germany \\ 4 European Southern Observatory, Karl-Schwarzschild-Str. 2, 85748 Garching, Germany \\ 5 Astronomical Institute "Anton Pannekoek", Kruislaan 400, 1098 SJ Amsterdam, The Netherlands \\ 6 Institute of Astronomy of the Russian Academy of Sciences, 48 Pyatnitskaya Str., 109107 Moscow, Russia
}

Received 10 April 2001 / Accepted 14 August 2001

\begin{abstract}
We have started a large survey for radial velocity variations in white dwarfs (PI R. Napiwotzki) with the aim of finding close double degenerates, which could be precursor systems for SNe Ia. The UVES spectrograph at the ESO VLT is used to obtain high resolution spectra with good $S / N$. During this project 1500 white dwarfs will be observed. This unique data set will also allow to derive atmospheric parameters and masses for the largest sample of white dwarfs ever analyzed in a homogenous way. In this paper we present a catalog of objects and report results for the first sample of about 200 white dwarfs, many of which are spectroscopic confirmations of candidates from the HE, MCT, and EC surveys. Among the peculiar spectra we identify two new magnetic DA, one previously known magnetic DA, several DA with emission cores, in some cases due to a late-type companion, and two new DBA.
\end{abstract}

Key words. stars: white dwarfs - stars: supernovae: general

\section{Introduction}

Type Ia supernovae (SNe Ia) play a prominent role in the study of cosmic evolution. They are believed to be the most important producers of iron in the universe and their energy input into the ISM may be responsible for driving the hot gas out of galactic spheroids. The fact that they can be seen to large distances and that their light curves are fairly uniform makes them one of the best standard candles for distance determinations, both in the nearby universe to determine $H_{0}$ as well as at $z$ up to $\sim 1$ to determine the deceleration of the expansion of the universe, hence setting important constraints on the cosmological parameters $\Omega$ and $\Lambda$ (Riess et al. 1998; Perlmutter et al. 1999). Several large observing programs, both with

\footnotetext{
Send offprint requests to: D. Koester, e-mail: koester@astrophysik.uni-kiel.de

* Based on observations obtained at the Paranal Observatory of the European Southern Observatory for program 165.H0588 .

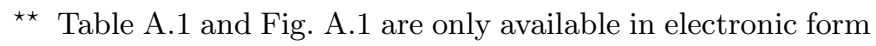
at http://www.edpsciences.org
}

HST and at all major ground-based observatories worldwide, are currently dedicated to searches for high redshift SNe Ia. They are likely to remain prime targets of astronomical investigations for years to come; they are, e.g., listed among the top priority targets for NGST (Next Generation Space Telescope) and OWL (Overwhelmingly Large Telescope), because with these instruments they could be observed all the way back to the re-ionization epoch.

Yet what kind of stars produce SN Ia events remains largely a mystery (Branch et al. 1995; Livio 1999). There is a general consensus that the event is due to the thermonuclear explosion of a white dwarf (WD) when a critical mass is reached, but the nature of the progenitor system remains unclear. It must be a binary system, with matter being transfered to the WD from a companion until the critical mass is reached. But there are still two main options for the nature of the companion: either another WD in the so-called double degenerate (DD) scenario (Iben \& Tutukov 1984), or a red giant/subgiant in the so-called single degenerate (SD) scenario (Whelan \& Iben 1973), with the system possibly appearing as a symbiotic 
binary (Munari \& Renzini 1992) or a supersoft X-ray source (van den Heuvel et al. 1992).

The solution of the SD vs. DD question is of great importance for the role of SN Ia as accurate cosmological probes, because it would constrain the possible explosion models. If the progenitors are indeed DDs, then one expects that the exploding objects will span a range of masses, depending on the sum of the two WDs in the binary. This would affect the light curves, and possibly the spectral evolution, and hints for such effects have been detected in the amount of nickel produced in SN Ia events (Mazzali et al. 1998; Contardo et al. 2000; Leibundgut 2000).

Several systematic radial velocity searches for DDs have been undertaken, starting in the mid 1980's (Robinson \& Shafter 1987; Bragaglia et al. 1990; Saffer et al. 1998; Maxted \& Marsh 1999). Quite a few DDs were indeed discovered and their periods determined, but none of the systems qualifies as a SN Ia progenitor, because the combined mass of the WDs is smaller than the Chandrasekhar limit. This is not surprising, as theoretical simulations suggest that perhaps only a few percent of all DDs are in a system with supercritical mass (Iben et al. 1997; Nelemans et al. 2001).

By now - combining all searches - about 150 WDs have been checked for RV variations with sufficient accuracy, which has led to the discovery of 15 DDs with period $P<6.3$ days (Marsh 2000). We have set ourselves the ambitious goal of increasing this sample by a factor of ten, with a Large Programmme (PI R. Napiwotzki) at the Very Large Telescope (VLT) of the European Southern Observatory at Paranal. The observations of two spectra at different times has already yielded more than 30 new DDs. These observations and the analysis of radial velocity curves will be published in separate papers. However, as a by-product of this project we obtain high-quality, high-resolution spectra with uniform observation and reduction methods for a large number of WDs, 1500 when the whole project will be completed. This is a homogeneous database of enormous value for many other areas of research (e.g. mass distribution of white dwarfs, kinematics, surface composition, luminosity function). We have therefore decided to make these results available to the community in the form of a catalog with some preliminary interpretation as soon as feasible, not waiting for the completion of the SNeIa progenitor project. This paper presents the first installment of this catalog, covering observations of about 200 white dwarfs of spectral types DA and DB.

\section{Observations and data reduction}

The candidates for this first sample were drawn from 4 sources: the white dwarf catalog of McCook \& Sion (1999), the Montreal-Cambridge-Tololo (MCT) survey (Lamontagne et al. 2000), the Edinburgh-Cape (EC) survey (Kilkenny et al. 1997), the Hamburg/ESO Survey
(Wisotzki et al. 1996; Wisotzki et al. 2000; Christlieb et al. 2001), and the Hamburg Quasar Survey (Hagen et al. 1995; Homeier et al. 1998). The selection criteria were spectroscopic confirmation as white dwarf (at least from objective prism spectra) and $B<16.5$.

Spectra were taken with the UV-Visual Echelle Spectrograph (UVES) of the Unit 2 Telescope (UT2 = Kueyen). UVES is a high-resolution Echelle spectrograph, which can reach a resolution of 110000 in the red region with a narrow slit (see Dekker et al. 2000 for a description of the instrument). Our instrument setup (Dichroic 1, central wavelengths $3900 \AA$ and $5640 \AA$ ) uses UVES in a dichroic mode with a $2048 \times 4096 \mathrm{EEV}$ CCD windowed to $2048 \times 3000$ in the blue arm, and two CCDs, a $2048 \times 4096 \mathrm{EEV}$ and a $2048 \times 4096$ MIT-LL, in the red arm. Nearly complete spectral coverage from $3200 \AA$ to $6650 \AA$ with only two $\approx 80 \AA$ wide gaps at $4580 \AA$ and $5640 \AA$ is achieved.

In the standard setting used for our observations UVES is operated with an $8^{\prime \prime}$ decker in the blue arm and an $11^{\prime \prime}$ decker in the red arm. This makes a reliable background subtraction possible. However, when we checked the spectra for signs of background contamination by moon light - e.g. the G band - we found some contamination, mostly in spectra taken under very unfavorable conditions. When a second, uncontaminated, spectrum is available, we have tested the influence of this background contamination on the derived parameters, and found it to be negligible.

Our program is implemented as a low-priority service mode program. It takes advantage of those observing conditions, which are not usable by most other programs (moon, bad seeing, clouds), and therefore it is sometimes qualified as a "filler program", to keep the VLT busy when other (more important) programs are not feasible. A wide slit $\left(2.1^{\prime \prime}\right)$ is used to minimize slit losses and a $2 \times 2$ binning is applied to the CCDs to reduce read out noise. Our wide slit reduced the spectral resolution to $R=18500(0.36 \AA$ at $\mathrm{H} \alpha$ ) or better, if the seeing disks were smaller than the slit width. Due to the nature of our project, two spectra at different epochs were taken. Depending on the brightness of the objects, exposure times of $5 \mathrm{~min}$ or $10 \mathrm{~min}$ were chosen. The $S / N$ per binned pixel $(0.05 \AA)$ of the extracted spectrum is usually 15 or higher. This restricts the brightness of our program stars to $B<16.5$.

Although our program is carried out during periods of less favorable observing conditions, the seeing is often smaller than the selected slit width of $2.1^{\prime \prime}$. This can, in principle, cause wavelength shifts, if the star is not placed in the center of the slit. Though not relevant for the analysis presented in this paper, we note that this can be corrected with the telluric absorption features present in the red region of our spectra.

The spectra were reduced with the ESO pipeline for UVES, based on MIDAS procedures. The raw data are bias and interorder background subtracted and extracted with an optimum extraction algorithm. The orders are flatfielded, rebinned, and merged. Wavelength 

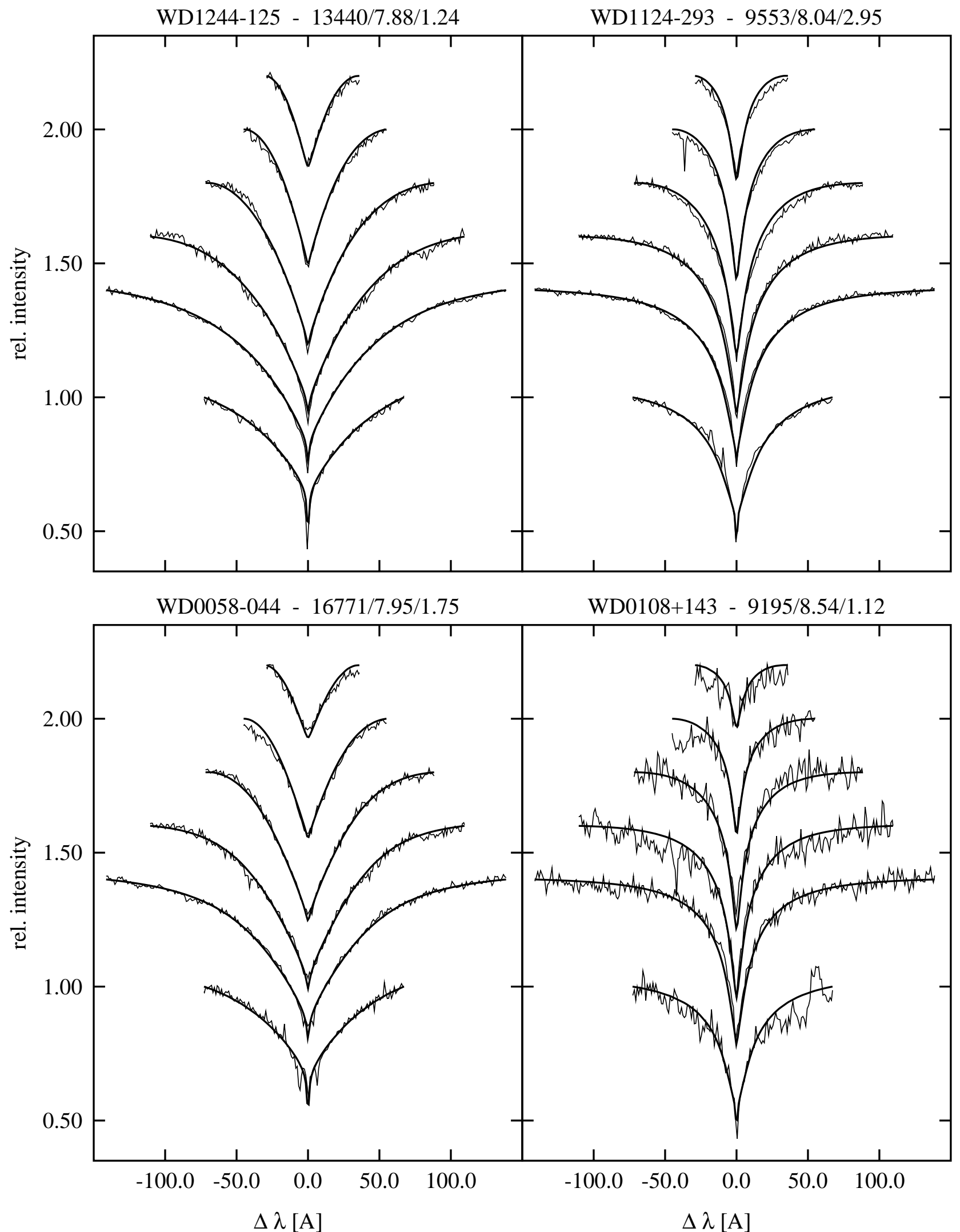

Fig. 1. Four examples of the fitting procedure for DA spectra, with Balmer lines H $\alpha$ to H8 from bottom to top. The header above the panels gives the name of the object, the effective temperature, surface gravity, and $\chi^{2}$ of the final fit. The figures are produced directly from the fitting routine; of course not all the digits in the temperature value e.g. are significant. The scale of relative intensities is correct for $\mathrm{H} \alpha$; the following lines are displaced upward by $0.4,0.2,0.2,0.2,0.2$ respectively. See text for more explanations. 


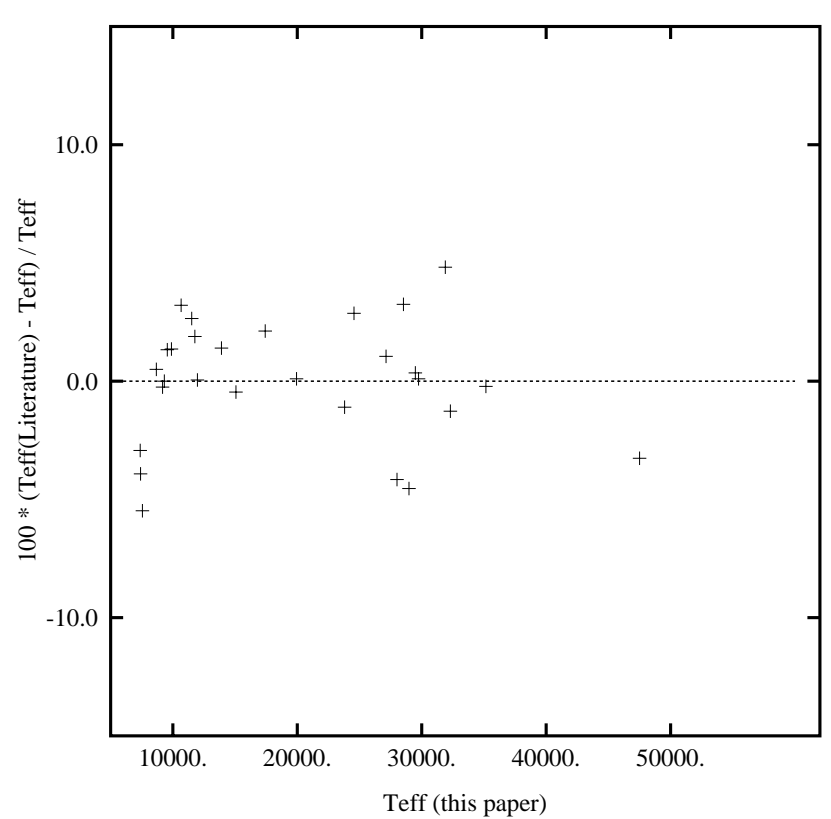

Fig. 2. Comparison of $T_{\text {eff }}$ determinations with values from the literature based on long-slit spectra or IR photometry.

calibration is performed by means of ThAr calibration lamp exposures. The quality of the reduced spectra is in most cases very good; especially the removal of the intraorder sensitivity variation and merging of the orders worked very well. Sometimes the reduction pipeline produced artefacts of varying strength, e.g. a quasiperiodic pattern in the red region similar in appearance to a fringing pattern. In a few cases either the blue or the red part of the spectrum had extremely strong artefacts of unknown origin, and these spectra were removed from the dataset and will be re-reduced in Bamberg.

Remaining large scale variations of the spectral response function were removed by utilizing nearly featureless spectra of DC white dwarfs or of sdO stars with narrow spectral lines, which were normalized and used to derive an approximate response function. This function was applied to the other spectra. This procedure worked very well in most spectral regions, with minor remaining problems e.g. in the $\mathrm{H} \delta$ region of a few objects (see WD1124-293 in Fig. 1). Since the files containing the reduced spectra are quite large and the sampling is much higher than needed for an analysis of the broad hydrogen and helium lines, we rebinned the spectra to $0.1 \AA$ stepsize and smoothed them with a Gaussian of $0.2 \AA F W H M$. This produces only a slight degradation of the resolution while considerably reducing the noise.

\section{Analysis of high resolution spectra and results}

The main aim of this paper is to present a quick-look physical interpretation, very similar in philosophy and implementation to the pipeline reduction of the data at ESO. The intention of the project is of course to search for double degenerates, and in the beginning we did not expect the spectra to be useful for a detailed spectral analysis.

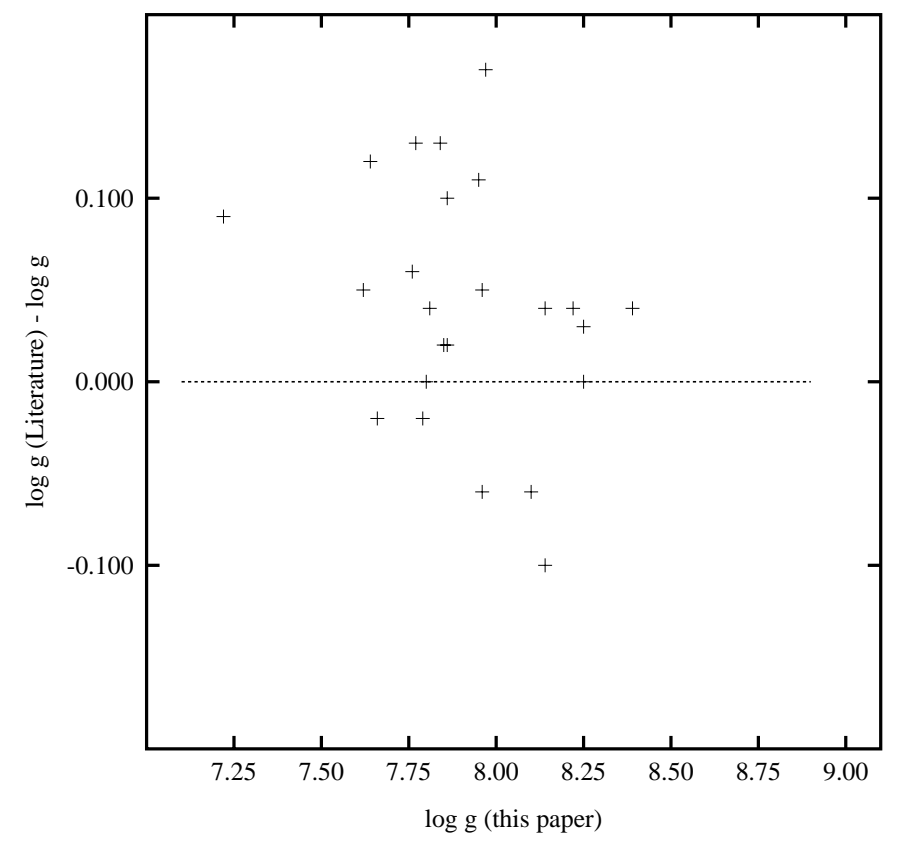

Fig. 3. Comparison of $\log g$ determinations with values from the literature based on long-slit spectra or IR photometry + parallaxes.

That turned out to be wrong, and one purpose of this work is to alert the community to the data of this project, which will all be available very soon in the VLT archive. It is important to note that a significant fraction of the objects - and an even larger one in the following papers - are new white dwarf identifications, mainly from the Hamburg ESO Quasar Survey (HE). We do not have the manpower to really exploit all the information in these data. Several of the objects show metal lines, some of them probably stellar - this is interesting material for the study of the accretion/diffusion scenario in DA white dwarfs. A more careful analysis could be done, adapting the spectral fitting for each individual spectrum to the highest quality spectral range of the observations, to get an accurate mass distribution. In the near term a study of the white dwarf kinematics is carried out in Bamberg, which will use the masses from this work to determine the velocity correction corresponding to the gravitational redshift. For all these reasons and constraints we have decided to use the data almost exactly as they come from the UVES reduction pipeline and implement the analysis in an analogous way: the original data go into the input end of the analysis pipeline, are merged, rebinned, rescaled, fitted with models and the fits plotted without human intervention. At the end, however, the final plots are visually inspected, any peculiarities noted, and spectra, which are not useful, are taken out of the queue, before a repeated run of the software produces the final results and output. This is all very similar to the philosophy of the reduction pipeline for the data at ESO.

Let us look at this analysis pipeline in some detail. As a first step for the spectral analysis the three different spectral ranges are further binned to approximately $1 \AA$ 
resolution and combined to one file per observation. This serves to improve the signal-to-noise ratio, but also to decrease the large amount of data to a more manageable size. The spectra are then fitted with theoretical spectra from a large grid of LTE DA and DB models, using a $\chi^{2}$ technique based on the Levenberg-Marquardt algorithm (Press et al. 1992). The input physics for our models is similar to the description in Finley et al. (1997); some details on the fitting method can also be found in Homeier et al. (1998).

Figure 1 shows four rather arbitrarily selected examples of this procedure. Theoretical spectra are fitted to the continuum on both sides of the Balmer lines and the best fit is then determined from the line profiles. The figure shows in the upper panels two DA spectra with good $S / N$; the right spectrum also has a CaII K line in absorption. The star in the lower left panel shows clear Zeeman splitting in the lower Balmer lines and is thus a magnetic DA. The lower right panel finally is an example for spectra with lower $S / N$.

Because the strength of the Balmer lines in white dwarfs reaches a maximum around $T_{\text {eff }}=12000 \mathrm{~K}$, it is often possible to find two minima with the $\chi^{2}$ minimization. We have always used two starting values for the iterative solution ( $9000 \mathrm{~K}$ and $15000 \mathrm{~K}$ ). If the solutions did not converge to a single value, we preferred the one with the lower $\chi^{2}$ value, which was further confirmed by a visual inspection.

Several of the DA, for which the LTE fit resulted in a temperature hotter than $40000 \mathrm{~K}$ were reanalyzed using a NLTE DA grid, to check the dependence of the results on possible NLTE effects. The models and fit procedure are described in Napiwotzki et al. (1999). The differences compared to the LTE fits were minor as expected; the tables in the following nevertheless give the NLTE results in these cases. One of the DA in this range (EC13123-2523) shows the so-called "Balmer line problem" (see Napiwotzki \& Rauch 1994): higher Balmer lines point to higher effective temperatures than the low lines and the overall fit with pure hydrogen models is poor. The problem has been traced back to the influence of EUV metal line blanketing (Werner 1996).

\subsection{White dwarfs of spectral type $D A$}

Table A.1 (Appendix, only available online) gives the results for the normal DA spectra. The objects are sorted by right ascension; the table gives the WD numbers from the white dwarf catalog (McCook \& Sion 1999), where further information can be found. For objects not in the catalog we attempt to give the designation from the discovery survey: HE for the Hamburg/ESO Quasar survey, EC for the Edinburgh-Cape Survey, and MCT for the Montreal-Cambridge-Tololo Survey; these papers are also the sources for the magnitudes. All coordinates were measured on the DSS (Digital Sky Survey) frames, and in many cases corrected for proper motion to epoch 2000.
Table 1. Comparison of echelle spectra with single-order spectra. The first line for each object gives the fit result from the echelle spectra of this work (in the case of two available echelle spectra the better one was used). The second line is a fit to lower resolution single-order spectra, using the same line intervals for fitting, except for $\mathrm{H} \alpha$, which is not available for the single-order spectra.

\begin{tabular}{|l|r|r|}
\hline object & $T_{\text {eff }}$ & $\log g$ \\
\hline WD0133-116 & 11768 & 7.84 \\
& 12301 & 8.03 \\
WD0302+027 & 36158 & 7.66 \\
& 38352 & 7.68 \\
WD1422+095 & 12700 & 7.95 \\
& 12847 & 7.84 \\
WD1544-377 & 10613 & 8.12 \\
& 11023 & 8.19 \\
WD1736+052 & 9064 & 8.24 \\
& 8783 & 8.19 \\
WD2014-575 & 28013 & 7.80 \\
& 28264 & 7.93 \\
WD2326+049 & 11515 & 7.97 \\
& 11969 & 8.20 \\
\hline
\end{tabular}

For objects, which already have two independent observations, we give both spectral fit results. The errors for the effective temperature and surface gravity are formal errors from the $\chi^{2}$ fitting routine, they do not include systematic errors and therefore usually underestimate the true error. A detailed discussion of realistic error limits for state-of-the-art analysis of DA white dwarfs is provided in Napiwotzki et al. (1999). The $\chi^{2}$ value for the best fit should not be over-interpreted, but only used as a relative measure of the fit quality; it depends on the noise of the spectrum, which is determined by filtering the continuum between the lines with a Savitzki-Golay filter and comparing the spectrum with the smoothed continuum. A spectrum with high $S / N$ may thus often have very small errors $(\sigma)$; if there are systematic differences between model and observation (as opposed to statistical), the minimum $\chi^{2}$ value will be large. The same is true, if e.g. imperfect background subtraction leaves large residuals of night sky lines or other artefacts. On the other hand, if the noise of the spectrum is large, the minimum $\chi^{2}$ may be fairly small and less influenced by systematic errors.

Detailed inspection of the fits shows that typically a $\chi^{2}$ value larger than about 2.5 indicates that the fit is not very good. This may be due to calibration problems, artefacts in the spectra that were introduced - or not removed - by the pipeline reduction, or the presence of e.g. He lines in the observations, which are of course not included in the models of our DA grid.

A more realistic determination of the parameter errors can be obtained using the two independent spectra available for many objects. For 81 normal DA with two independent determinations we find an average difference of $500 \mathrm{~K}$ for $T_{\text {eff }}$ and $0.08 \operatorname{dex}$ for $\log g$. The absolute error in $T_{\text {eff }}$ is larger for the hotter DA, a reasonable estimate for all objects is to assume a one $\sigma$ error for $T_{\text {eff }}$ of about $3 \%$. 

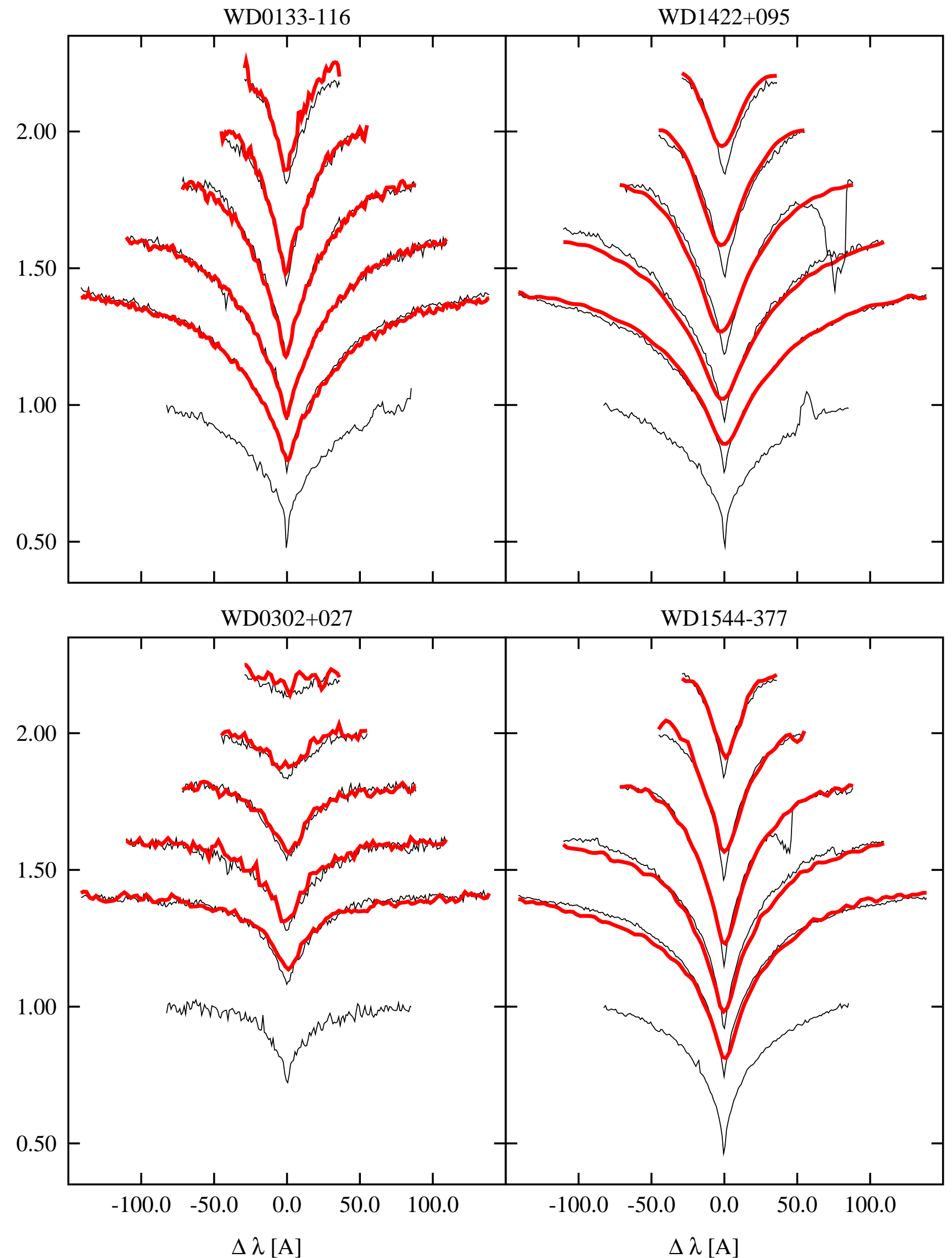

Fig. 4. Comparison of DA echelle spectra (thin line) with single-order spectra of lower resolution (thick line) from various telescopes and instruments. 


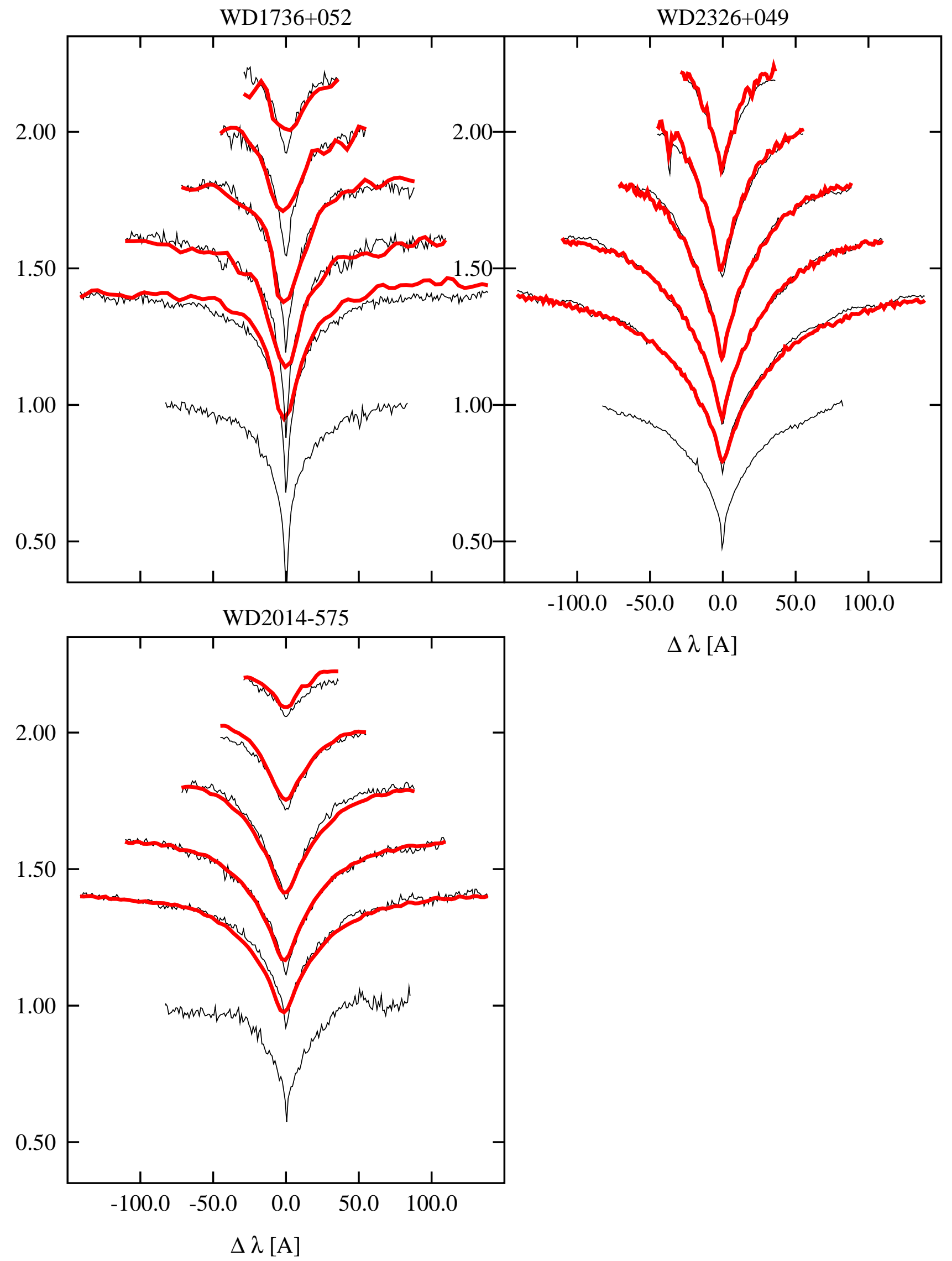

Fig. 5. Comparison of echelle and single-order spectra for 3 more DA. 
Table 2. Peculiar objects with $\mathrm{H}$ lines. Columns contain from left to right the object name, right ascension and declination, a magnitude (usually $V, B$ stands for photographic $B$ magnitude, $v$ for multichannel $v$ magnitude), effective temperature and its formal error, surface gravity and formal error, and a remark.

\begin{tabular}{|l|r|r|l|r|r|r|r|l|}
\hline object & $\alpha(2000)$ & $\delta(2000)$ & $V$ & $T_{\text {eff }}$ & $\Delta T_{\text {eff }}$ & $\log g$ & $\Delta \log g$ & $\operatorname{Rem}$ \\
\hline WD0058-044 & $01: 01: 02.3$ & $-04: 11: 11$ & 15.38 & 16700 & 62 & 8.07 & 0.01 & 1 \\
WD0128-387 & $01: 30: 28.0$ & $-38: 30: 39$ & 15.32 & 27909 & 135 & 8.54 & 0.02 & 2 \\
WD0131-163 & $01: 34: 24.1$ & $-16: 07: 08$ & 13.98 & 57508 & 1014 & 8.17 & 0.05 & 3 \\
WD0239+109 & $02: 42: 08.5$ & $+11: 12: 32$ & 16.18 & 46859 & 569 & 7.69 & 0.05 & 4 \\
HE0331-3541 & $03: 33: 52.5$ & $-35: 31: 19$ & $14.8 \mathrm{~B}$ & 31372 & 343 & 7.70 & 0.08 & 5 \\
WD0347-137 & $03: 50: 14.6$ & $-13: 35: 14$ & $14.00 \mathrm{~B}$ & 21296 & 331 & 8.27 & 0.05 & 5 \\
HE1103-0049 & $11: 06: 27.7$ & $-01: 05: 15$ & 16.2 & 30607 & 186 & 7.37 & 0.04 & 6 \\
WD1247-176 & $12: 50: 22.1$ & $-17: 54: 48$ & 16.19 & 20922 & 317 & 8.06 & 0.05 & 5 \\
WD1319-288 & $13: 22: 40.5$ & $-29: 05: 35$ & 15.99 & 18012 & 212 & 7.77 & 0.04 & 5 \\
EC13349-3237 & $13: 37: 50.8$ & $-32: 52: 23$ & 16.34 & 48116 & 1353 & 6.99 & 0.10 & 5 \\
HE1346-0632 & $13: 48: 48.3$ & $-06: 47: 21$ & 16.2 & 30194 & 301 & 6.94 & 0.06 & 7 \\
WD1350-090 & $13: 53: 15.6$ & $-09: 16: 33$ & $14.55 \mathrm{v}$ & 23794 & 140 & 7.34 & 0.02 & 8 \\
WD2211-495 & $22: 14: 11.9$ & $-49: 19: 27$ & 11.70 & 63983 & 891 & 7.06 & 0.04 & 9 \\
\hline
\end{tabular}

Remarks: 1: new magnetic DA: Zeeman triplet $\mathrm{H} \alpha$ : 6556.827/6563.044/6569.301 А̊, H $\beta$ also with triplet; 2: DAB (McCook \& Sion 1999); 3: several Balmer line cores with emission, H $\alpha$ possibly multiple peaks; 4: G4-34, called "probable DA+DB unresolved binary" by Bergeron et al. (1990); it is clearly magnetic with Zeeman splitting in $\mathrm{H} \alpha$ to $\mathrm{H} \delta$; $\mathrm{H} \alpha$ : 6548.215/6562.455/6576.422; splitting may be variable; 5: Balmer lines with emission cores; red continuum; DA+dM binary; 6: H $\alpha$ with emission core, CaII in emission; 7: redshifted emission component in Balmer lines; 8: known magnetic (Schmidt \& Smith 1994); triplet in all Balmer lines; $\mathrm{H} \alpha$ 6555.514/6564.646/6573.778 $\AA$; 9: emission core in $\mathrm{H} \alpha$ and $\mathrm{H} \beta$.

Table 3. Observed DB and parameters from spectral fitting. The next-to-last column gives the effective temperature obtained assuming $\log g=8$. See Table 2 for further explanations of the other entries.

\begin{tabular}{|c|c|c|c|c|c|c|c|c|c|}
\hline object & $\alpha(2000)$ & $\delta(2000)$ & $V$ & $T_{\text {eff }}$ & $\Delta T_{\text {eff }}$ & $\log g$ & $\Delta \log g$ & $T_{\text {eff }}(\log g=8)$ & Rem \\
\hline HE0025-0317 & $00: 27: 41.7$ & $-03: 00: 58$ & $15.7 \mathrm{~B}$ & 19602 & 280 & 8.59 & 0.06 & 17356 & 1 \\
\hline WD0119-004 & $01: 21: 48.3$ & $-00: 10: 54$ & $16.00 \mathrm{~B}$ & 16285 & 85 & 8.25 & 0.05 & 15900 & \\
\hline WD0119-004 & & & & 16375 & 99 & 8.24 & 0.06 & 16107 & \\
\hline WD0300-013 & 03:02:53.2 & $-01: 08: 35$ & 15.56 & & & & & 14930 & 2 \\
\hline HE0417-5357 & 04:19:10.0 & $-53: 50: 46$ & $15.1 \mathrm{~B}$ & 18785 & 50 & 8.13 & 0.02 & 18563 & \\
\hline HE0417-5357 & & & & 18773 & 62 & 8.24 & 0.02 & 17668 & \\
\hline HE0420-4748 & $04: 22: 11.4$ & $-47: 41: 42$ & $14.7 \mathrm{~B}$ & 24285 & 204 & 8.16 & 0.02 & 24641 & \\
\hline HE0420-4748 & & & & 25176 & 252 & 8.16 & 0.03 & 25436 & \\
\hline WD0615-591 & $06: 16: 14.5$ & $-59: 12: 28$ & 14.09 & 16874 & 94 & 8.22 & 0.04 & 16885 & \\
\hline WD1149-133 & 11:51:50.6 & $-13: 37: 15$ & 16.29 & 18607 & 120 & 8.43 & 0.04 & 17207 & 3 \\
\hline WD1149-133 & & & & 19542 & 152 & 8.45 & 0.03 & 17487 & 3 \\
\hline EC12438-1346 & $12: 46: 30.4$ & $-14: 02: 41$ & 16.39 & 17640 & 81 & 8.28 & 0.04 & 17027 & \\
\hline WD1336+123 & 13:39:13.6 & $+12: 08: 30$ & 13.90B & 16869 & 70 & 8.26 & 0.03 & 16448 & \\
\hline HE1349-2305 & $13: 52: 44.3$ & $-23: 20: 07$ & 16.3 & 16770 & 113 & 7.95 & 0.05 & 16939 & \\
\hline WD1428-125 & $14: 31: 39.6$ & $-12: 48: 56$ & 15.98 & 20452 & 194 & 8.39 & 0.03 & 19205 & \\
\hline WD1428-125 & & & & 20365 & 196 & 8.40 & 0.03 & 19203 & \\
\hline WD1444-096 & $14: 47: 37.0$ & $-09: 50: 06$ & 14.98 & 17417 & 100 & 8.39 & 0.04 & 16639 & \\
\hline WD2316-173 & $23: 19: 35.4$ & $-17: 05: 29$ & 14.04 & & & & & 11008 & 4 \\
\hline WD2316-173 & & & & & & & & 12639 & 4 \\
\hline
\end{tabular}

Remarks: 1 : DBA with strong $\mathrm{H} \beta$ and $\mathrm{H} \gamma ; 2$ : strong CaII; no fit with variable $\log g$; 3 : DBA with weak $\mathrm{H} \beta 4$ : DBQA4 according to McCook \& Sion (1999).

Another possibility is the comparison with parameter determinations based on long-slit spectra (since the echelle spectra also use a long slit, we will call this singleorder spectra further-on) or optical and infrared photometry available in the literature for about 30 of our objects. This comparison is shown in Figs. 2 and 3. For $T_{\text {eff }}$ the systematic shift is about $0.6 \%$, for $\log g 0.03$ dex; given the larger differences between determinations by different authors even using only high quality single-order spectra (Napiwotzki et al. 1999) this is clearly not significant. The scatter in both diagrams confirms the estimates of parameter uncertainties given above.

A more direct comparison of the combination of echelle reduction effects and the analysis is possible for $7 \mathrm{DA}$, for 


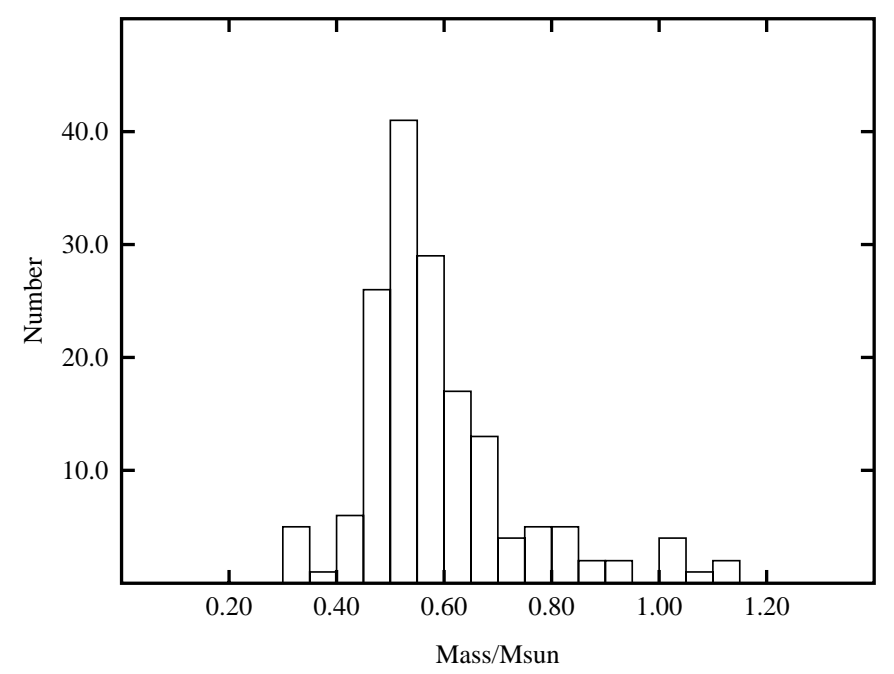

Fig. 6. Mass distribution of the DA white dwarfs in Table A.1.

which we have single-order spectra with lower resolution available from different telescopes and instruments, gathered for various programs over the last 15 years at La Silla and Calar Alto. Figures 4 and 5 display the Balmer line profiles of these objects in a similar way as in Fig. 1, except that the second curve is not a model, but the lower resolution single-order spectrum. The lower resolution is most obvious in the different central line depths; there are also small wavelength shifts, and some noticeable differences also in the line wings. However, in general the agreement between echelle and single-order spectra is remarkably good. This is also demonstrated by a comparison fit to the single-order spectra, using as far as possible the same spectral intervals as for the echelle spectra. Effective temperatures and surface gravities are compared in Table 1; considering the fact that the single-order spectra are from many different sources and not necessarily of better quality than the echelle spectra, the comparison seems satisfactory, and confirms that the reduction and analysis procedures should produce results of an accuracy comparable to that for traditional single-order spectral analysis.

For those readers interested in individual objects from Table A.1 or in a general assessment of the quality of the fits we have provided in Appendix A a graphical representation of the line fits for all objects in Table A.1 (only available in the online version of the paper).

\subsection{Properties of the DA sample}

A statistical analysis of the DA observed in this project will be presented, when the observations of the whole sample ( 1500 objects) is complete. For a very preliminary analysis we have determined individual masses for all "ordinary" DA in Table A.1 with good determinations of atmospheric parameters, using the evolutionary massradius relation of Wood (1995) for "thick" hydrogen envelope masses $\left(10^{-4}\right.$ of the stellar mass). The values are also given in the table; the resulting mass distribution for
163 DA confirms the expectations based on several largescale studies of DA white dwarfs during the last decade based on high $S / N$ low-resolution spectra or optical and infrared photometry, e.g. Bergeron et al. (1992); Bragaglia et al. (1995); Finley et al. (1997); Bergeron et al. (1997); Napiwotzki et al. (1999). The average surface gravity is 7.89 , with a one $\sigma$ width of the distribution of 0.32 . The average mass is $0.59 M_{\odot}$, with a one $\sigma$ width of $0.15 M_{\odot}$. The mass distribution is plotted in Fig. 6, it shows the typical structure known from many previous studies: the peak between 0.45 and $0.60 M_{\odot}$ containing the majority of DA, the secondary peak below $0.45 M_{\odot}$, that both theory and observation ascribe to helium-core WDs resulting from binary evolution (Bragaglia et al. 1990; Marsh et al. 1995; Yungelson et al. 2000), and a tail at large masses above $1.0 M_{\odot}$.

\subsection{Peculiar spectra with hydrogen lines}

A few objects show peculiar line profiles. These are summarized in Table 2. In some cases the Balmer lines have emission cores, which may be due to NLTE effects for $T_{\text {eff }}$ larger than $40000 \mathrm{~K}$, or to the presence of a late-type companion. Three objects are magnetic DA and show Zeeman splitting of $\mathrm{H} \alpha$ and sometimes higher lines as well.

\subsection{White dwarfs of spectral type $D B$}

Our sample also contains a number of white dwarfs of spectral type DB. These were fitted in a way very similar to the DA sample, using a grid of DB model spectra, which employs the recent line broadening calculations of Beauchamp et al. (1997). As in the DA, there are often two solutions possible: one above and one below the temperature of maximum line strengths, which is about $20000 \mathrm{~K}$ in DB. We have used two different starting values $(15000$ and $25000 \mathrm{~K}$ ) for the iteration. As for the DA, if the routine converged on two different solutions, we have selected the correct one from the $\chi^{2}$ value and visual inspection. The results for the parameters are summarized in Table 3, Fig. 7 shows three examples for the spectra and model fits. Similar figures on a smaller scale for all objects in Table 3 are also given in Appendix A (online version only).

It is generally more difficult to determine surface gravities for DB than for DA. Our results seem to be unusually high, with only one value below $\log g=8.0$. The average mass - determined with the Wood (1995) relation for "thin layers", appropriate for DB - is $0.77 M_{\odot}$, significantly higher than for the DA. This is in contradiction to previous studies (e.g. Oke et al. 1984; Beauchamp et al. 1999). Our model atmospheres use a composition of pure helium; about $20 \%$ of the DB show detectable traces of hydrogen and a small admixture of hydrogen cannot be excluded in other DB, which might affect the determination of atmospheric parameters (Beauchamp et al. 1999). We have therefore repeated the fit with a model grid with 

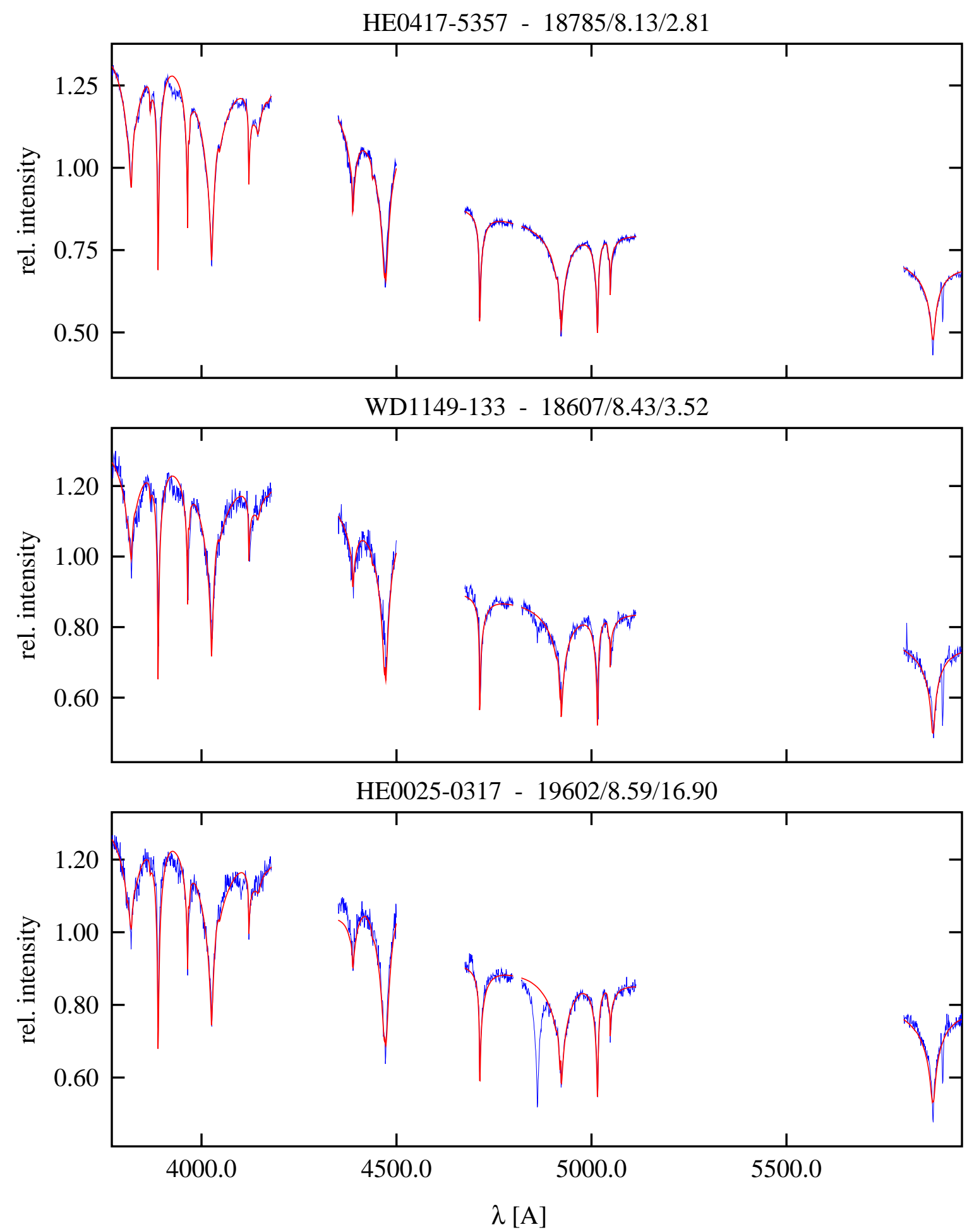

Fig. 7. Typical spectra and model fits for helium-rich objects. From top to bottom: a DB with high $S / N$, a DBA with lower quality spectrum and a weak $\mathrm{H} \beta$ line, and a DBA with strong $\mathrm{H} \beta$ and visible $\mathrm{H} \delta$. Over most of the spectral range the models (thinner lines) fit the spectra within the noise and can hardly be distinguished.

a $\mathrm{H} / \mathrm{He}$ ratio of $10^{-5}$, which results in even slightly larger surface gravities.

We have currently no explanation for this result, and do not know, whether it is real or somehow an artefact of the reduction of the echelle spectra, especially the normalization and merging of the orders. The DA spectra seem to give very reasonable results, although the individual lines are broader. However, since in the DB case many lines overlap, the wavelength regions, which are fitted in one piece are broader than for the DA. We have only one classical single-order spectrum for one of the DB (WD1428-125 = HE1428-1235) of our current 

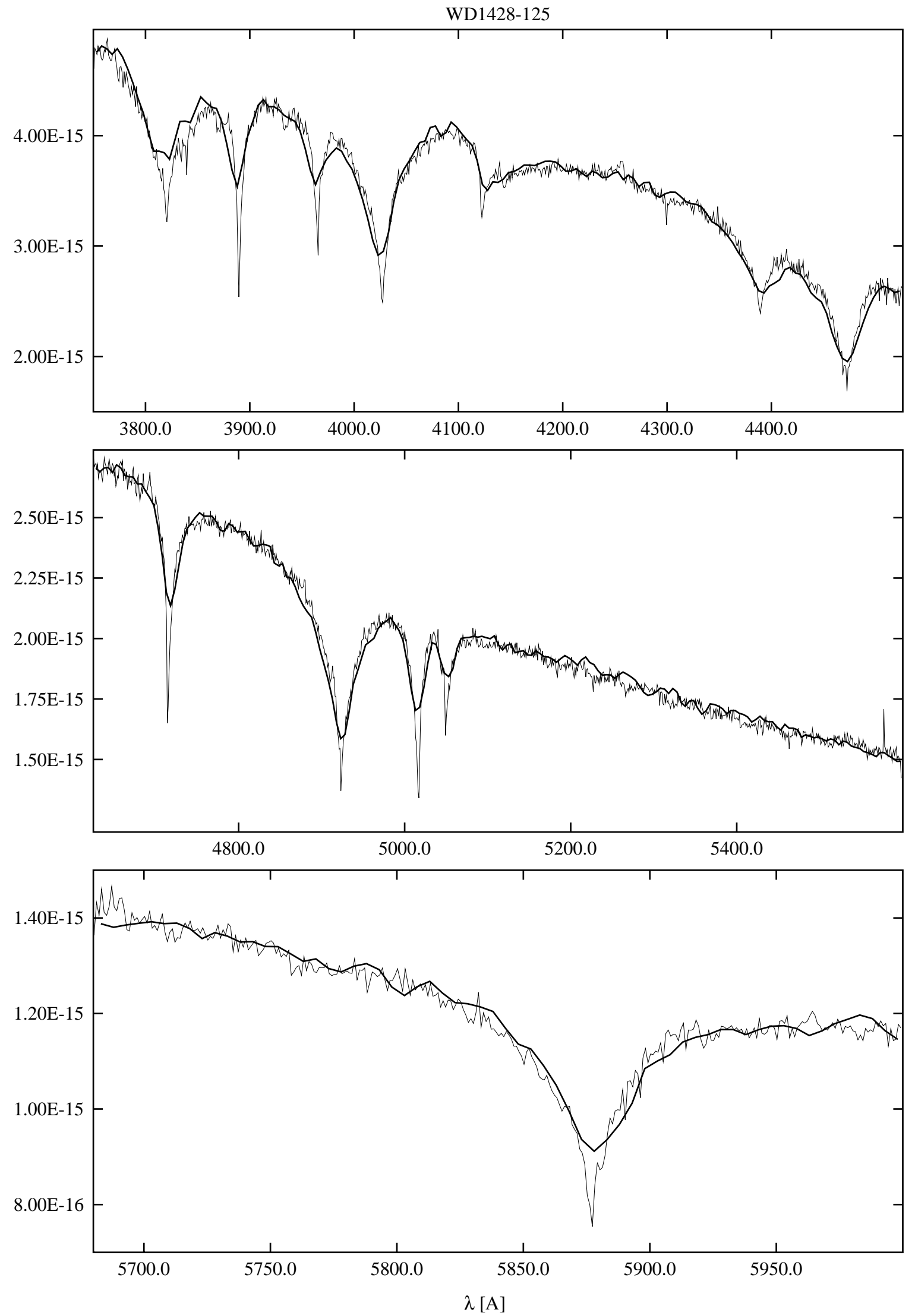

Fig. 8. Comparison of echelle (thin line) and single-order spectra for the DB white dwarf WD1428-125 in 3 spectral regions, approximately corresponding to regions used for the spectral fitting with models. 
sample at our disposal. A comparison of the single-order with the echelle spectra is shown in Fig. 8 for 3 wavelength regions. The echelle spectra in this case have been fitted to the single-order spectrum at the continuum points used for fitting the models in our DB analysis procedure. The agreement of the line profiles is similar as for the DA and does not show any obvious discrepancies. This spectrum has been studied in a detailed analysis by Friedrich et al. (2000), who find $T_{\text {eff }}=19050 \mathrm{~K}, \log g=7.87$, as compared to our values of $20450 / 8.39$. While most of the differences in the line profiles in Fig. 8 are due to the very different resolution ( 15 vs. $1 \AA$ ), there are obviously other small differences not compensated for by the $\chi^{2}$ fitting routine, which bias the solution towards higher $\log g$ values. Because of this uncertainty, we have included in the table also a fit with surface gravity fixed to 8.00 for all objects.

\section{Conclusions}

Our study confirms that the echelle spectra - even with only the UVES pipeline reduction applied - show well calibrated line profiles, which can be used to determine atmospheric parameters with the same accuracy and reliability as from lower resolution spectroscopy without echelle grating (single-order spectra). This was not necessarily expected, since the Balmer lines in the DA span several echelle orders. The very large and homogenous sample of spectra, which will in the end be obtained for this program will thus be of great value in addition to the original purpose of searching for double-degenerate SNe Ia precursors for the determination of mass distributions and kinematic properties of white dwarfs. The mass determinations of this work are a prerequisite for the determination of space velocities, where the radial component has to be corrected for the individual gravitational redshifts.

Acknowledgements. We want to express our gratitude to the ESO staff, for providing invaluable help and conducting the service observations and pipeline reductions, which have made this work possible. D.K. and D.H. thank the Deutsche Forschungsgemeinschaft (DFG) for their support (Ko739/10-3). C.K. and E.M.P. also acknowledge support by the DFG (Na365/2-1). S.M. was supported by a grant (50 OR 96029-ZA) from the Bundesministerium für Bildung und Forschung through the DLR. L.Y. is supported by RFBR grant 99-02-16037 and Program "Astronomy" grant 1.4.4.1.

\section{Appendix A: Observed DA white dwarfs and parameters from spectral fitting}

In Table A.1 we give data for the observed DA white dwarfs and the parameters derived from the spectral fitting procedure. This table is made available only electronically. It contains columns with the following information object name

$\alpha(2000)$;

$\delta(2000)$; visual or $B$ magnitude;

effective temperature from spectral fit;

formal error $(1 \sigma)$ for $T_{\text {eff }}$;

surface gravity $\log g$;

formal error $(1 \sigma)$ for $\log g$;

$\chi^{2}$ for the fit;

mass of the white dwarf in solar masses; remarks.

\section{Appendix B: Graphical display of model fits for the DA in Table A.1 and the DB in Table 3}

In Fig. A.1, which is only available in electronic form, we present the model fits for the normal DA and DB in the same form as in Figs. 1 and 7. Unfortunately, the scale has to be very small, and sometimes for the good fits it is hard to distinguish model and observation. A postscript file with larger scale and using different colors for model and observation is available upon request from D. Koester.

\section{References}

Beauchamp, A., Wesemael, F., \& Bergeron, P. 1997, ApJS, 108,559

Beauchamp, A., Wesemael, F., Bergeron, P., et al. 1999, ApJ, 516, 887

Bergeron, P., Liebert, J., \& Greenstein, J. L. 1990, ApJ, 361, 190

Bergeron, P., Ruiz, M. T., \& Leggett, S. K. 1997, ApJS, 108, 339

Bergeron, P., Saffer, R. A., \& Liebert, J. 1992, ApJ, 394, 228

Bergeron, P., Wesemael, F., Fontaine, G., \& Liebert, J. 1989, ApJ, 345, L91

Bragaglia, A., Greggio, L., Renzini, A., \& D’Odorico, S. 1990, ApJ, 365, L13

Bragaglia, A., Renzini, A., \& Bergeron, P. 1995, ApJ, 443, 735

Branch, D., Livio, M., Yungelson, L. R., Boffi, F. R., \& Baron, E. 1995, PASP, 107, 1019

Christlieb, N., Wisotzki, L., Reimers, D., et al. 2001, A\&A, 366,898

Contardo, G., Leibundgut, B., \& Vacca, W. D. 2000, A\&A, 359,876

Dekker, H., D'Odorico, S., Kaufer, A., Delabre, B., \& Kotzlowski, H. 2000, Proc. SPIE, 4008, 534

Finley, D. S., Koester, D., \& Basri, G. 1997, ApJ, 488, 375

Friedrich, S., Koester, D., Christlieb, N., Reimers, D., \& Wisotzki, L. 2000, A\&A, 363, 1040

Hagen, H.-J., Groote, D., Engels, D., \& Reimers, D. 1995, A\&AS, 111, 195

Homeier, D., Koester, D., Hagen, H. J., et al. 1998, A\&A, 338, 563

Iben, I., \& Tutukov, A. V. 1984, ApJ, 282, 615

Iben, I. J., Tutukov A. V., \& Yungelson L. R. 1997, ApJ, 475, 291

Jordan, S., Heber, U., Engels, D., \& Koester, D. 1993, A\&A, 273, L27

Kilkenny, D., O’Donoghue, D., Koen, C., Stobie, R. S., \& Chen, A. 1997, MNRAS, 287, 867

Lamontagne, R., Demers, S., Wesemael, F., Fontaine, G., \& Irwin, M. J. 2000, AJ, 119, 241

Leibundgut, B. 2000, A\&AR, 10, 179 
Livio, M. 1999, in Annapolis Workshop on Magnetic Cataclysmic Variables, ed. C. Hellier, \& K. Mukai, ASP Conf. Ser., 157, 247

Marsh, T. R. 2000, New. Astr. Rev., 44, 119

Marsh, T. R., Dhillon, V. S., \& Duck, S. R. 1995, MNRAS, 275,828

Maxted, P. F. L., \& Marsh, T. R. 1999, MNRAS, 307, 122

Mazzali, P. A., Cappellaro, E., Danziger, I. J., Turatto, M., \& Benetti, S. 1998, ApJ, 499, L49

McCook, G. P., \& Sion, E. M. 1999, ApJS, 121, 1

Munari, U., \& Renzini, A. 1992, ApJ, 397, L87

Napiwotzki, R., Green, P. J., \& Saffer, R. A. 1999, ApJ, 517, 399

Napiwotzki, R., \& Rauch, T. 1994, A\&A, 285, 603

Nelemans, G., Yungelson, L. R., Portegies Zwart, S. F., \& Verbunt, F. 2001, A\&A, 365, 491

Oke, J. B., Weidemann, V., \& Koester, D. 1984, ApJ, 281, 276

Perlmutter, S., Aldering, G., Goldhaber, G., et al. 1999, The Supernova Cosmology Project 1999, ApJ, 517, 565

Press, W. H., Teukolsky, S. A., Vetterling, W. T., \& Flannery, B. P. 1992, Numerical recipes in FORTRAN, The art of scientific computing (Cambridge: University Press), -c1992, 2nd Ed.
Riess, A. G., Filippenko, A. V., Challis, P., et al. 1998, AJ, 116, 1009

Robinson, E. L., \& Shafter, A. W. 1987, ApJ, 322, 296

Saffer, R. A., Livio, M., \& Yungelson, L. R. 1998, ApJ, 502, 394

Schmidt, G. D., \& Smith, P. S. 1994, ApJ, 423, L63

van den Heuvel, E. P. J., Bhattacharya, D., Nomoto, K., \& Rappaport, S. A. 1992, A\&A, 262, 97

Werner, K. 1996, A\&A, 309, 861

Whelan, J., \& Iben, I. J. 1973, ApJ, 186, 1007

Wisotzki, L., Christlieb, N., Bade, N., et al. 2000, A\&A, 358, 77

Wisotzki, L., Koehler, T., Groote, D., \& Reimers, D. 1996, A\&AS, 115, 227

Wood, M. A. 1995, in White Dwarfs, Proceedings of the 9th European Workshop on White Dwarfs Held at Kiel, Germany, 29 August - 1 September 1994, XII, 348, ed. D. Koester, \& K. Werner (Springer-Verlag, Berlin, Heidelberg, New York), Also Lect. Notes Phys., 443, 51

Yungelson, L. R., Nelemans, G., Portegies Zwart, S. F., \& Verbunt, F. 2000, preprint [astro-ph/0011248] 